\title{
Application of Accelerometer Data to Atmospheric Modeling During Mars Aerobraking Operations
}

\author{
R. H. Tolson* \\ North Carolina State University, Hampton, Virginia 23666-6147 \\ G. M. Keating \\ George Washington University, Newport News, Virginia 23602 \\ R. W. Zurek主 \\ Jet Propulsion Laboratory, California Institute of Technology, Pasadena, California 91109-8099 \\ S. W. Bougher $\stackrel{\S}{-}$ \\ University of Michigan, Ann Arbor, Michigan 48109-2143 \\ C. G. Justus $\mathbb{I}$ \\ Morgan Research Corporation, Huntsville, Alabama 35805-1948 \\ and \\ D. C. Fritts** \\ NorthWest Research Associates, Inc., Boulder, Colorado 80301
}

\section{DOI: $10.2514 / 1.28472$}

\begin{abstract}
This paper reviews the use of accelerometer data for determining atmospheric density during aerobraking operations of the Mars Global Survey, Mars Odyssey, and Mars Reconnaissance Orbiter missions. For all three missions, accelerometer data were analyzed in near real time to provide estimates of thermospheric density, density scale height, latitudinal gradients, global longitudinal wave structure, and small-scale (gravity) wave spectra. Mars Global Survey, Mars Odyssey, and Mars Reconnaissance Orbiter provided data during approximately 850, 320, and 425 passes covering latitude ranges of $60^{\circ} \mathrm{N}$ to $90^{\circ} \mathrm{S}, 30^{\circ} \mathrm{N}$ to $90^{\circ} \mathrm{N}$, and 0 to $90^{\circ} \mathrm{S}$, respectively. Periapsis altitudes varied from 95 to $130 \mathrm{~km}$. A summary is given of the atmospheric phenomena encountered during the aerobraking phase of the missions and of some of the scientific results based on these data.
\end{abstract}

\section{Nomenclature}

$\mathrm{L}_{s}=$ longitude of the mean sun along the Mars equator

$\rho=$ density, $\mathrm{kg} / \mathrm{km}^{3}$

$\sigma=$ standard deviation

\section{Introduction}

A EROBRAKING (AB) is the use of atmospheric drag for beneficial orbit changes via multiple passes through an upper atmosphere. The first planetary application of aerobraking from which atmospheric properties were recovered was in 1993 during the Venus Magellan mission [1] , in which aerobraking was performed for 70 days and for approximately 750 orbital passes. Because accelerometer data were not available during that mission, atmospheric density [2] at periapsis was recovered by the more traditional method of determination of the change in the orbital period from tracking data. During Magellan cycle 4, when periapsis was between

Presented as Paper 6397 at the AAIAA/AAS Astrodynamics Specialist Conference and Exhibit, Keystone, CO, 21-24 August 2006; received 22 October 2006; revision received 29 March 2007; accepted for publication 10 May 2007. Copyright @ 2007 by the American Institute of Aeronautics and Astronautics, Inc. The U.S. Government has a royalty-free license to exercise all rights under the copyright claimed herein for Governmental purposes. All other rights are reserved by the copyright owner. Copies of this paper may be made for personal or internal use, on condition that the copier pay the $\$ 10.00$ per-copy fee to the Copyright Clearance Center, Inc., 222 Rosewood Drive, Danvers, MA 01923; include the code 0022-4650/07 $\$ 10.00$ in correspondence with the CCC.

*Professor, 100 Exploration Way. Associate Member AIAA.

${ }^{\dagger}$ Senior Research Staff Scientist, 1 Old Oyster Point Road, Suite 200. Associate Member AIAA.

*Mars Reconnaissance Orbiter Project Scientist, Mail Stop 264-535.

\$Professor, Space Physics Research Laboratory, 2455 Hayward Avenue.

"Senior Principal Scientist, 4811-A Bradford Drive.

${ }^{* *}$ Colorado Research Associates Division, 3380 Mitchell Lane.
165 and $185 \mathrm{~km}$, spacecraft attitude data were used to estimate density and scale height $[3,4]$, with very good agreement with the drag results. The second $\mathrm{AB}$ mission began in September 1997 and ended with more than 850 Mars Global Surveyor (MGS) AB passes [5,6]. Mars Odyssey (ODY) followed in October 2001 with over 300 $\overline{\mathrm{AB}}$ passes [7]. Mars Reconnaissance Orbiter (MRO) began $\mathrm{AB}$ in April 2006 and ended in August 2006 with 425 AB passes [8,9]. All of these Mars mission established frozen orbits for the science phase with 93-deg inclinations. The spacecraft were equipped with three axis accelerometers with measurement noise below $0.5 \mathrm{~mm} / \mathrm{s}$ for a 1 -s count and allowed density recovery to better than $1 \mathrm{~kg} / \mathrm{km}^{3}$. For reference, aerobraking corridors are usually defined in terms of maximum freestream heat flux, resulting in nominal densities of about $30 \mathrm{~kg} / \mathrm{km}^{3}$ at periapsis. MGS (ODY) provided meaningful density data up to about $160(150) \mathrm{km}$. The noise level for the much improved MRO accelerometer is below $0.005 \mathrm{~mm} / \mathrm{s}^{2}$, equivalent to a density of about $0.01 \mathrm{~kg} / \mathrm{km}^{3}$.

As seen in Fig. 1, periapsis locations for these missions cover a significant fraction of the seasonal $\left(\mathrm{L}_{s}\right)$, diurnal [local solar time (LST)], and latitudinal space of interest for validation of atmospheric models. Phase 1 (G1) of MGS AB was between 30 and $60^{\circ} \mathrm{N}$ during fall $\left(\mathrm{L}_{s}=180^{\circ}\right)$ and early winter $\left(\mathrm{L}_{s}=270^{\circ}\right)$. MGS phase $2(\mathrm{G} 2)$ started at $60^{\circ} \mathrm{N}$ and arrived at the south pole as winter $\left(\mathrm{L}_{s}=90^{\circ}\right)$ began. ODY $(\mathrm{O})$ completed coverage of the north pole winter. MRO (R) provided similar coverage to MGS at the south pole and provided the first opportunity to use past experience during AB operations. As seen in the lower right panel, aerobraking altitude generally decreases as periapsis approaches the pole, because the atmosphere is cooling and constant density surfaces decrease in altitude. In the three-dimensional cube, these mission curves leave many voids, but in the two-dimensional projections, the southern hemisphere springsummer quadrant remains the most obvious unsampled region. The opportunity exists to sample this region with a 2011-2012 aerobraking mission. The lack of coverage between LST of 6 and $12 \mathrm{~h}$ is due to the characteristic that interplanetary trajectories have 

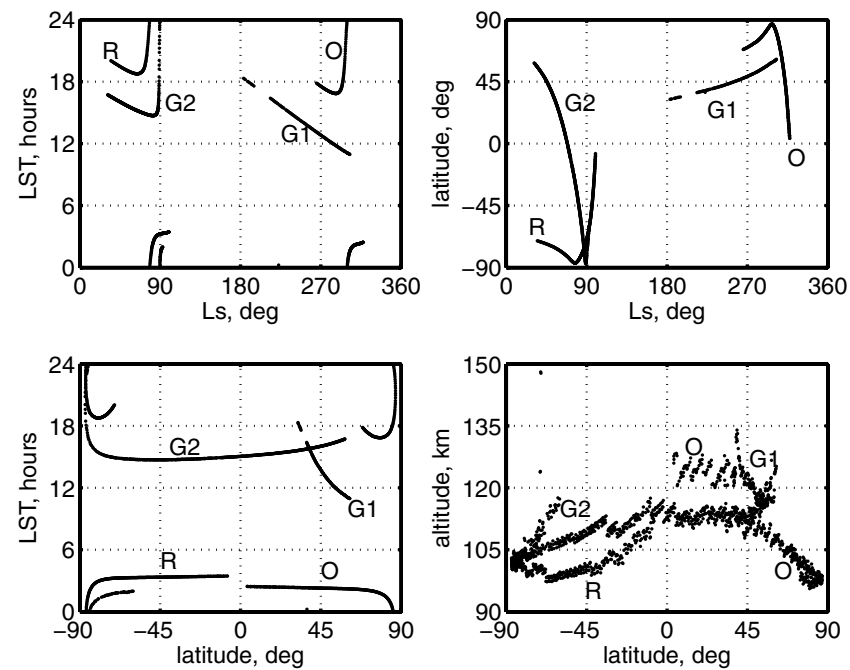

Fig. 1 Span of atmospheric parameters covered by MGS, ODY, and MRO aerobraking periapsis locations.

approach hyperbola periapsis between about $15 \mathrm{~h}$ (type I) and $21 \mathrm{~h}$ (type II).

\section{Small-Scale Variability}

It had been suggested before the MGS mission that the Martian thermosphere demonstrated between 30 and $40 \%$ short-time-scale variability and that small-scale gravity waves might propagate to aerobraking altitudes. Almost every $\mathrm{AB}$ pass confirms those suggestions.

Figure 2 provides evidence of significant wave structure during four typical ODY AB passes. The solid lines are the recovered density from the accelerometer data and the dashed lines are MarsGRAM [10,11] profiles that have been scaled to provide the same drag effect or approximately the same area under the profiles. During Odyssey, this scale factor was determined for each orbit and varied from 0.35 to 0.8 [12]. During MRO, the scale factor varied from 1 to 5.5. ODY orbits 155 and 157 were during a phase of the mission in which $\mathrm{AB}$ was in or near the polar vortex, which is a concentrated westerly jet near $60^{\circ}$ latitude near the winter pole. The outbound leg of orbit 155 shows the traditional bell shape and closely follows the scaled MarsGRAM profile. From 0 to $200 \mathrm{~s}$, the vehicle latitude is north of $82^{\circ} \mathrm{N}$ and the variability is typical of the low smallscale variability observed when inside the vortex. The "bump" in the inbound leg at $-100 \mathrm{~s}$ is about twice the outbound density at $+100 \mathrm{~s}$ and occurs at $75^{\circ} \mathrm{N}$ latitude. Periapsis occurs at about $82^{\circ} \mathrm{N}$ latitude and $270^{\circ}$ E longitude. Orbit 157 covers the same latitudes as orbit 155 but with periapsis at $162^{\circ} \mathrm{E}$ longitude. The respective periapsis altitudes are 101.2 and $102.3 \mathrm{~km}$ and the latitudes and LST are essentially the same. On both orbits, a maximum latitude of $87^{\circ} \mathrm{N}$ was reached about $100 \mathrm{~s}$ after periapsis. Derivation of a density scale height from accelerometer data is complicated by the combined response to both horizontal and vertical density gradients projected along the spacecraft trajectory during an $\mathrm{AB}$ pass. The density scale height at periapsis is about $10 \mathrm{~km}$. Based on altitude difference only, a $11 \%$ change in periapsis density would have been expected. Like those shown here, a factor-of-two change in maximum density from orbit to orbit occurred occasionally throughout all three missions.

The density scale height of $10 \mathrm{~km}$ in the lower thermosphere implies an atmospheric temperature between 140 and $150 \mathrm{~K}$, which is much warmer than expected or experienced on MGS. Postflight analysis showed a strong latitudinal temperature gradient increasing toward the pole, suggesting a polar warming. Reference [13] interprets this warming as due to strong southern hemisphere summer solar and dust heating near perihelion. This heating induces strong interhemispheric circulation and increased adiabatic heating near the north pole.

By P199, periapsis precessed farther south to $72^{\circ} \mathrm{N}$ and the latitude is north of $78^{\circ} \mathrm{N}$ from 75 to $400 \mathrm{~s}$. The inbound leg had five waves
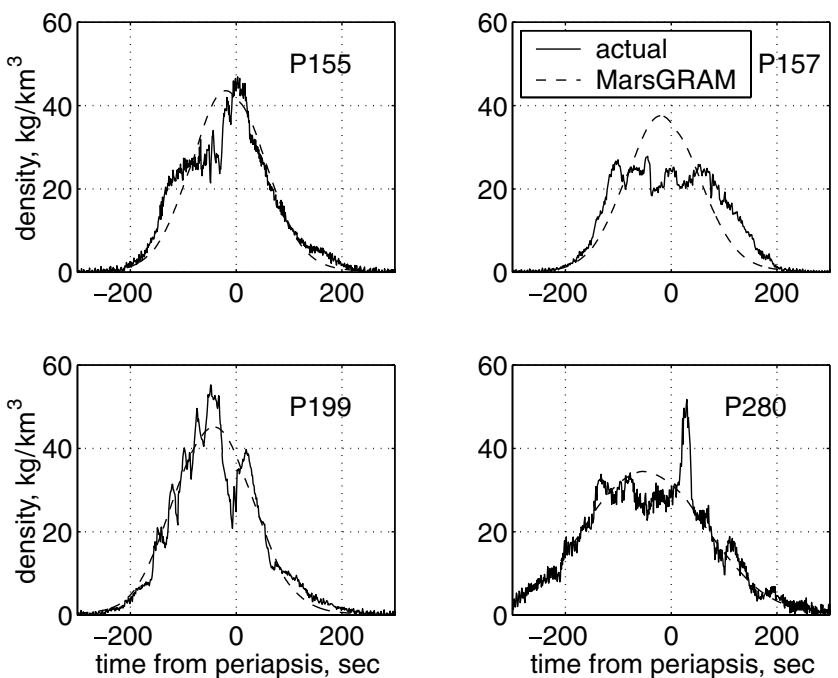

Fig. 2 Four Odyssey density histories. Periapsis latitudes are north at 82, 82, 71, and 46 deg $N$ and $L s$ is 298, 298, 302 and 307 deg.

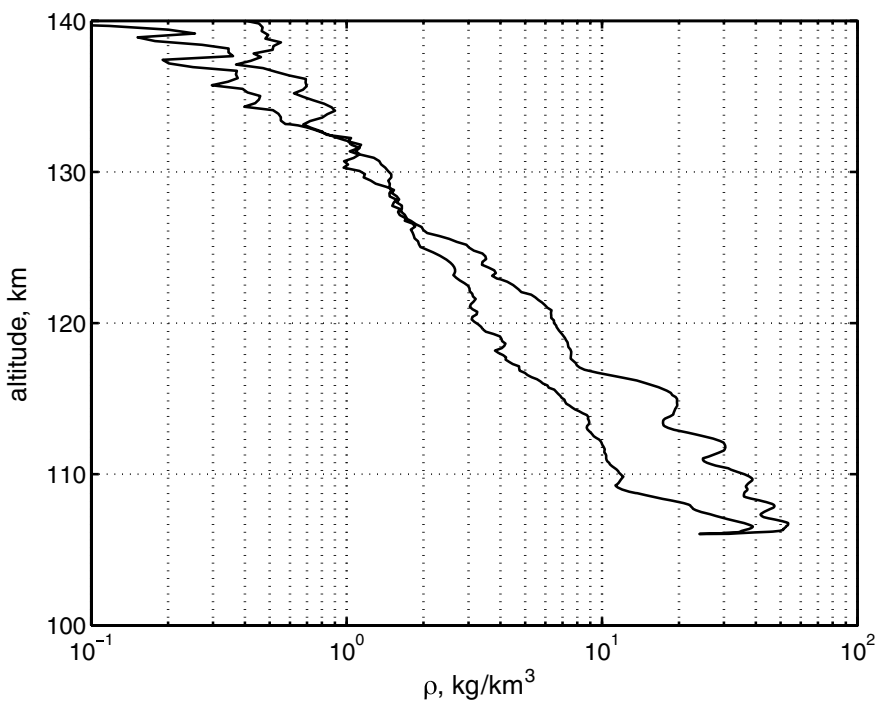

Fig. 3 P199 density profile after 7-s averaging. Altitude above reference ellipsoid.

above periapsis. For these waves, also shown vs altitude in Fig. 3 , the peak-to-trough density ratios vary between 1.2 and 1.9 and the peaks are about $2^{\circ}$ apart in latitude and increase from 1 to $3 \mathrm{~km}$ in vertical wavelength. The last peak just after periapsis is followed by a low variability outbound leg after $75 \mathrm{~s}$ at a latitude of $78^{\circ} \mathrm{N}$. The interpretation is that the vortex boundary is near $76^{\circ}$, giving a highly variable profile up to $75 \mathrm{~s}$ when outside the vortex and low variability inside the vortex after $75 \mathrm{~s}$. Just before periapsis there is more than a factor-of-2 decrease in density in about $10 \mathrm{~s}$ as the altitude decreased by less than $1 \mathrm{~km}$. Such rapid decreases are not uncommon and occasionally occur even over smaller altitude changes. In Fig. $\underline{3}$, minimal wave structure appears from 115 - to $130-\mathrm{km}$ altitude but may then start again above $130 \mathrm{~km}$. Somewhat similar structure above $130 \mathrm{~km}$ may suggest that the upper atmosphere waves have a large latitudinal span.

Finally, nearly circular orbit 280 is included to show that local phenomena can produce nearly factor-of- 2 changes in density over very short time scales. The latitude range is from $35^{\circ} \mathrm{N}$ at $-200 \mathrm{~s}$ to $57^{\circ} \mathrm{N}$ at $+200 \mathrm{~s}$. There is considerable variability from a mean profile on both the inbound and outbound legs. The spike just after periapsis has a latitude width of about $2^{\circ}$. It should be kept in mind that for these $\mathrm{AB}$ missions the solar array temperature has been the limiting factor and that thermal capacity and conduction through the solar array smooths many of these short-term variations and such 

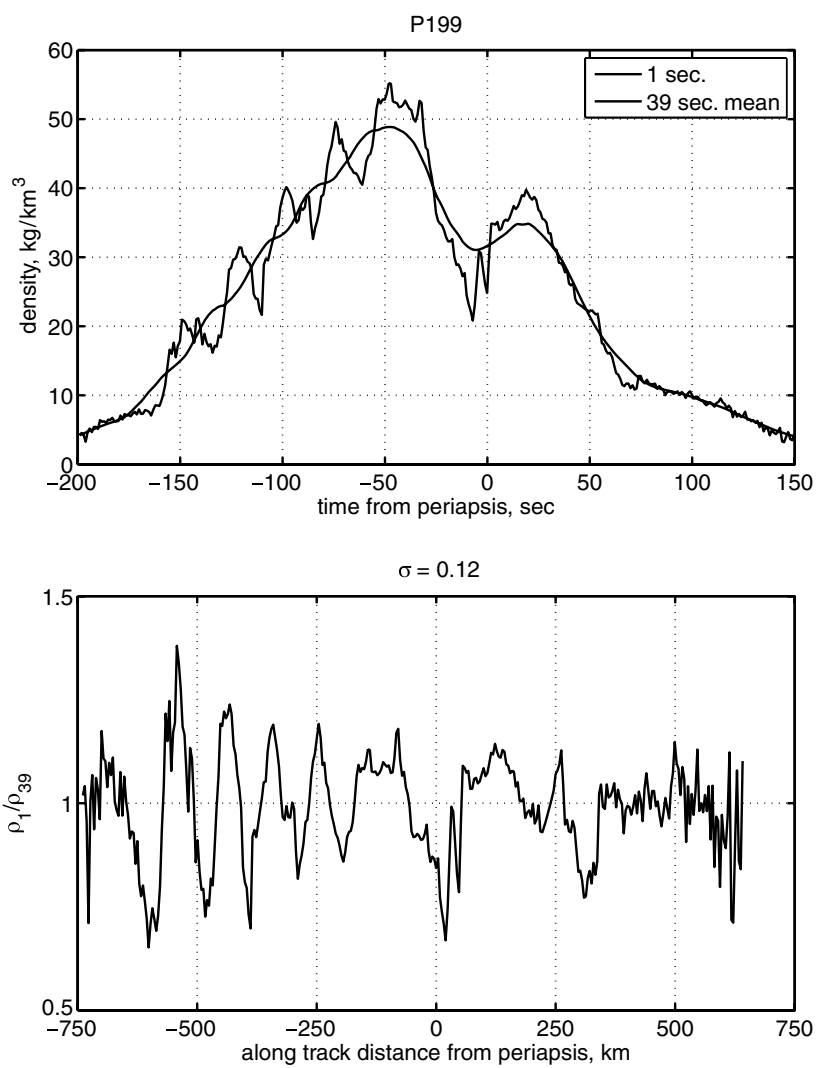

Fig. 4 P199 density history and ratio of 1-s samples to 39-s running mean.

local peaks may contribute little to the solar array maximum temperature.

The small-scale variations along an orbital pass have been generally attributed to gravity waves. Studies [14,15] have characterized the altitudinal, latitudinal, and seasonal variability and correlated these with topography and the mean jet. A first cut at the variability during each orbit is illustrated for orbit 199 in Fig. 4. The small-scale waves are extracted by comparison of the 1-s data with the 39-s running mean. The $s / c$ had a horizontal velocity of about $4 \mathrm{~km} / \mathrm{s}$, and so the averaging is over about $1600 \mathrm{~km}$ along track. From Fig. 3, the altitude range covered in Fig. 4 is from 105 to about $120 \mathrm{~km}$. The lower chart shows horizontal wavelengths of about $100 \mathrm{~km}$. Separating the vertical and horizontal structure is part of an ongoing analysis. Nevertheless, the standard deviation of this ratio of densities is a metric for gravity wave activity and orbit 199 had a standard deviation of 0.12 over the entire pass.

This type of analysis was performed on each orbit of MGS, Odyssey, and MRO, resulting in Fig. 5. The Odyssey data are only presented poleward of $55^{\circ} \mathrm{N}$, because there was a significant increase in accelerometer data noise during later times in the mission [7]. Periapsis is precessing toward the pole during the initial phases of all three missions (G1, O1, and R1) as well as late in MGS phase 2. The seasons are fall-winter and LST is between 12 and $24 \mathrm{~h}$ (Fig. 1). All three suggest a strong increase in variability as periapsis approaches $60^{\circ}$ latitude and a rapid drop near the winter pole. These increasing trends in variability have been interpreted as due to the presence of the winter jet permitting waves to propagate to higher altitudes [14]. For all missions, after periapsis passed over the pole and into the early morning hours, there is lower variability, perhaps associated with a more stable nighttime lower atmosphere. After midnight, MGS and ODY show a small increase as periapsis precesses equatorward, but MRO continually decreases, perhaps due to precession to a later morning hour (Fig. 1). MGS phase 1 (G1) and ODY, during precession toward the pole $(\mathrm{O} 1)$, have the highest variability. It was initially thought the large MGS values might be associated with the Noachis dust storm, but ODY and MRO have similar variability.
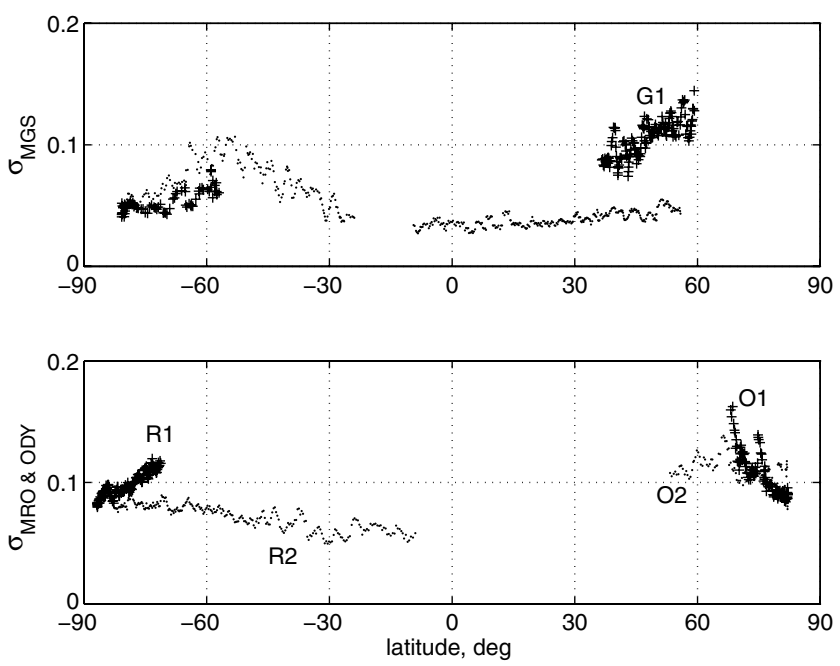

Fig. 5 MGS, ODY and MRO small-scale variability.

Though limited in latitudinal and seasonal range, studies [14] of the ODY variances, such as those shown in Fig. 4, led to a number of results. For orbits 7 to 134 , it was found that there is a localization of high variance in the 130 to $180^{\circ}$ longitude and $120-140-\mathrm{km}$ altitude ranges. Because of the limited sampling, it is difficult to separate spatial and temporal variations. Nevertheless, high spatial and temporal variability suggest filtering by large-scale waves or tides. Wave spectra exhibited consistent shape with altitude, but amplitude growth was much smaller than expected in the absence of dissipative processes. Gravity wave momentum flux estimates were much larger than comparable Earth fluxes, adding additional evidence that gravity waves have significant influence on large-scale motion. In [15], it is shown that thermospheric gravity waves variability is not geographically related to lower-atmosphere gravity wave source regions. For example, the tropics are a source region in the lower atmosphere and, as seen from Fig. 5, this is the region of lowest variability in the thermosphere.

\section{Orbit-to-Orbit Variability}

One of the thermospheric properties of interest during $\mathrm{AB}$ operations is the orbit-to-orbit variability. For short-period orbits, this variation is likely due to longitudinal waves, whereas for longperiod orbits, a combination of longitudinal and temporal variations likely contribute. To quantify this variation, a parameter called persistence is calculated using the density profile from one pass to predict the density at the periapsis altitude of the next pass. Because altitude, latitude, and LST change so little from pass to pass, an exponential density variation with altitude is fit by least squares to determine density at periapsis and the local scale height, which is typically between 6 and $10 \mathrm{~km}$. This simple model is used to predict the density at the next periapsis altitude. If no maneuvers are used to raise or lower periapsis, subsequent periapsis, perturbed primarily by the noncentral gravity field, is generally within $1.5-\mathrm{km}$ altitude. Of course, profiles such as that in Fig. $\underline{3}$ put into doubt the estimation of both periapsis density and density scale height.

Figure 6 shows the ratio of the observed density $\rho_{o}$ to density $\rho_{p}$ predicted from the previous orbit as a function of latitude. One orbit of ODY had a ratio of 3.3 and another of 4.2 , which are plotted as + at a ratio of 2.9. The whole-mission standard deviations of these persistence ratios $\sigma_{p}$ are $0.39,0.47$, and 0.36 for MGS, ODY, and MRO, respectively. References [7,16] demonstrate that the gamma probability distribution provides a reasonable model for wholemission persistence. With mean values of 1.06, 1.09, and 1.05, persistence is slightly more likely to underestimate than overestimate density. These data have borne out the preflight estimate of $40 \% 1 \sigma$ orbit-to-orbit change. However, there is significant latitudinal structure in the variability showing the highest ratios in the equatorial region and lowest ratios near the pole. For MGS orbits at latitudes within $30 \mathrm{deg}$ of the equator, $\sigma_{p}=0.54$, whereas poleward of $60^{\circ} \mathrm{S}$, 

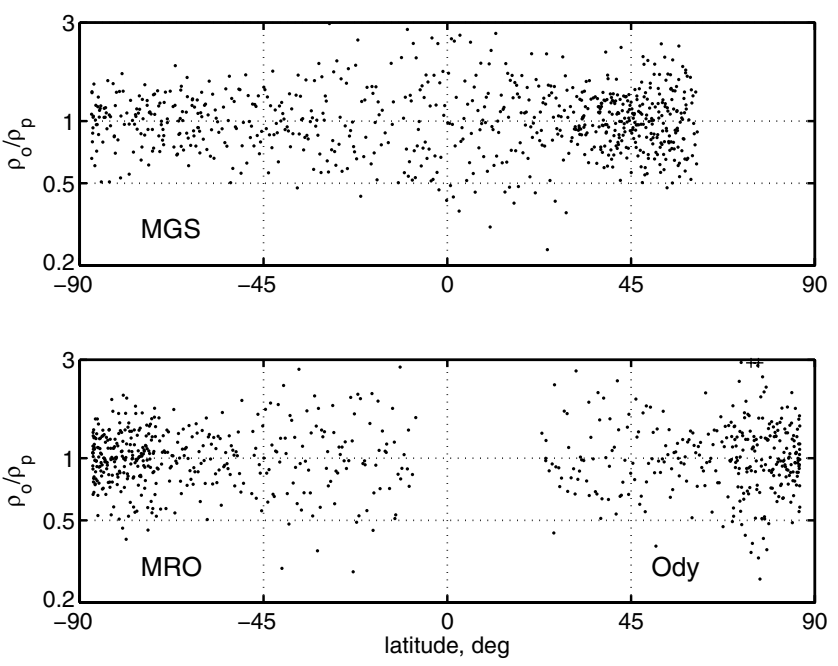

Fig. 6 MGS, ODY and MRO orbit-to-orbit persistence.

$\sigma_{p}=0.26$. MRO and ODY show minima near $60^{\circ}$ latitude, at which the small-scale variability (Fig. 5) is a maximum, suggesting that the polar jet plays a role in both small-scale and global wave propagation into the thermosphere.

For phase 1 of MGS, periapsis precesses from about 35 to $60^{\circ} \mathrm{N}$ (Fig. 1) during northern winter and $\sigma_{p}=0.38$ over the entire 155orbit interval. At the start of phase 2, periapsis precessed southward and from 60 to $30^{\circ} \mathrm{N}$ gave $\sigma_{p}=0.30$. The Noachis dust storm [17] occurred near orbit 50 , causing more than a $100 \%$ increase in density. On the 20 orbits before the storm, $\sigma_{p}=0.22$, and after the storm up to the end of phase $1, \sigma_{p}=0.39$. So in addition to doubling the density in the lower thermosphere, the storm appeared to also increase the variability. Though difficult to observe in the figure, variability decreased for periapses within about $10 \mathrm{deg}$ of the pole. Recall that all three missions perform polar crossing in the fall or winter, and so the interpretation has been that the polar vortex is blocking loweraltitude waves from propagating into this region.

For MGS, the increased variability in the tropics is largely due to global-scale stationary waves throughout this region [17], and Fig. 7 shows an example of these waves. Densities were determined at reference altitudes at $10-\mathrm{km}$ intervals. The + in the plots represents these data. The solid line is a stationary s-wave 4 fit to the data. The term s-wave will denote waves that are assumed to be stationary in longitude to distinguish them from the traditional atmospheric wave number notation. The dashed lines are the $1 \sigma$ deviation based on the formal statistics. At the lower altitude, nearest to where AB occurs, the maximum-to-minimum density ratio is about 3. During MGS and MRO operations, these types of models were used to improve prediction capability. No persistent wave structure was identified during ODY. It is seen that these s-waves maintain phase across the entire range of altitudes at which density can be extracted from the data. Shortly after the end of MGS AB, a model was developed for use in Mars Climate Orbiter AB design [16]. The model provides the amplitude and phase of s-waves 1 through $\overline{3}$ along the nearly constant density surface represented by MGS AB altitude. A significant increase in amplitude of s-wave 1 was also noted during the Noachis dust storm without a change in longitudinal phase. Longitude phase shifts due to either latitudinal or seasonal changes occurred rapidly for s-wave 2 and more slowly for s-wave 1 .

Although not recognized during MGS operations, the s-wave-2 component was anticipated preflight [18] as the result of the westward propagating wave 1 diurnal Kelvin wave. Because this Kelvin wave is in near resonance with the rotation of Mars, the wave appears nearly stationary to a sun-synchronous satellite. Because of the satellite being in a near-sun-synchronous orbit, other waves have also been attributed to lower-atmosphere nonmigrating tides that appear to be stationary $[19,20]$. The s-wave- 3 component is "largely due to a wave $2 \mathrm{~K}$ mode and an eastward-propagating wave 1 semidiurnal tide" [20]. A comparison of general circulation model (GCM)-derived density-wave-component amplitudes shows [19]

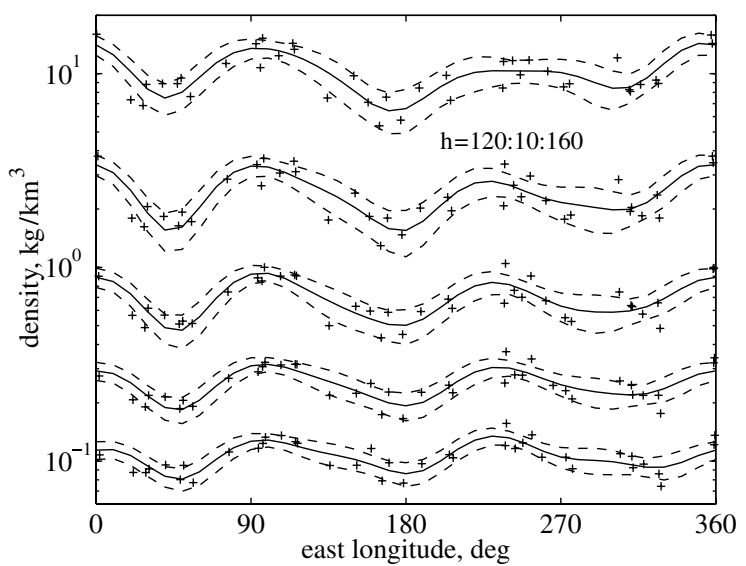

Fig. 7 Stationary waves derived from MGS orbits 587-626.
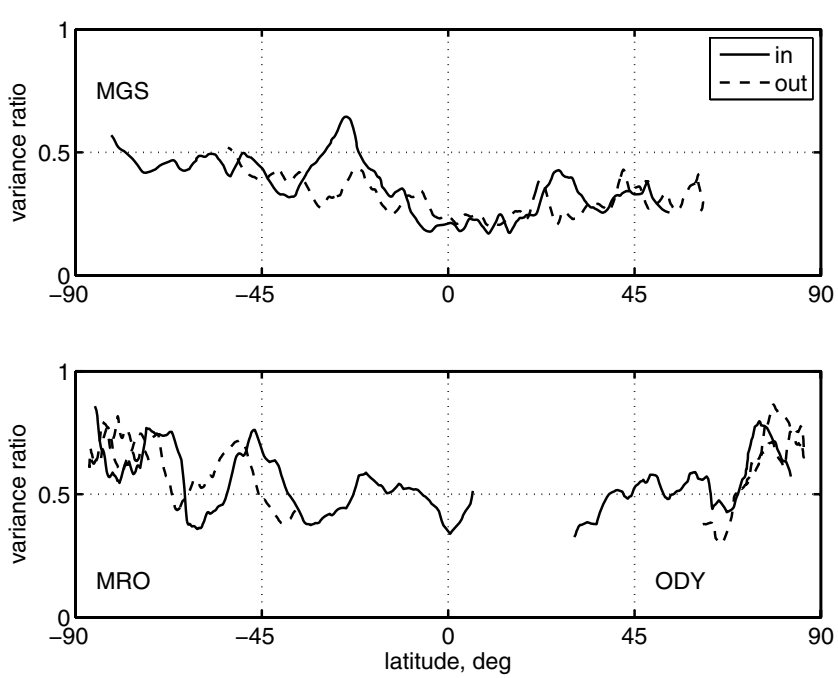

Fig. 8 Ratio of variances after and before wave 4 least squares running fit to density data at $125 \mathrm{~km}$.

very good agreement with accelerometer-derived density s-wave amplitudes.

A simple metric to identify the existence of s-waves is to perform a running fit of a density-wave model at a fixed altitude and compare the variance after the least-squares fit to the variance of the original data set. Figure 8 shows the ratio of these two variances when a wave4 model is used along with 41 orbits of data centered on each orbit. The inbound and outbound legs are fit independently. Note that orbital periods vary from 18 to $2 \mathrm{~h}$, and so this is probably not the optimal way to search for s-waves. During these three missions, the most persistent waves occurred during phase 2 of MGS, which begins at about $50^{\circ} \mathrm{N}$ in the upper panel and precesses to the south pole. It is seen that the variance ratio is less than 0.5 over almost all of this phase. During ODY operations, repeated attempts to use wavemodel predictions showed little promise. This was especially important early in the mission when periapsis was near the pole. But it can be see that there is little reduction in variance for any mission in the polar regions. Postflight analysis [7] suggests that a weak drifting wave 1 was present that was not captured by the constant-phase models. There were periods of time during MRO when the wave model showed promise. So even though both missions show increased orbit-to-orbit variability (Fig. 6) in the tropics, neither provided the persistent stationary waves demonstrated by phase 2 of MGS. Reference [16] provides amplitude and phase variations for swaves 1 through 3 for the entire MGS mission.

\section{Noachis Dust Storm}

Following the 22-orbit hiatus to recover from the discovery of the broken solar array, MGS started aerobraking again on orbit 40 . 
Thirteen orbits later, the Noachis regional dust storm occurred and the density at periapsis increased by more than $100 \%$. Over the rest of MGS phase 1, the density seemed to exponentially decay back to expected values. After the storm was recognized, periapsis altitude was immediately raised, resulting in $140 \mathrm{~km}$ being the lowest reference altitude with complete coverage over this time. Figure 9 shows the inbound and outbound density measurements after the hiatus. Inbound (outbound) latitude starts at $44^{\circ} \mathrm{N}\left(27^{\circ} \mathrm{N}\right)$ and precesses to $69^{\circ} \mathrm{N}\left(52^{\circ} \mathrm{N}\right)$. A model was fit to these data that assumed density varied quadratically with latitude over the entire data set and exponentially with time after orbit 52 . The solid line shows the resulting model prediction and the dashed line is only the latitudinal contribution. The best fit occurs for a decay time of 50 days for both the inbound and outbound data, but the solution is equally good for decay times between 45 and 55 days. Density more than doubles from the mean before orbit 53 to the model prediction at orbit 53 . Orbit-to-orbit variability has also significantly increased and, for the inbound leg, decays along with the density perturbation. Outbound orbit-to-orbit variability also decays, but not as rapidly as inbound. The inbound latitudes are more northerly, and the higher decay rate could be due to the dust storm decay as well as to the decrease with latitude shown in Fig. 6 .

\section{Crosswind Recovery}

The inherent aerodynamic stability of $\mathrm{AB}$ vehicles provides the opportunity to recover winds that influence either the pitch (vertical winds) or yaw (crosswinds) of the vehicle. Along-track winds are too small, relative to the vehicle velocity of $4 \mathrm{~km} / \mathrm{s}$, to be recovered. MGS and ODY performed AB with the solar arrays nearly horizontal so that the vehicle orientation is most sensitive to a crosswind. Preliminary wind recovery has been reported for MGS [21]. All three missions have orbits with 93-deg inclinations; thus, except near the pole, the cross-track component is essentially the zonal wind throughout most of the orbit. During an AB pass, both MGS and ODY weather vane into the apparent wind. Deviations from the trim orientation produce aerodynamic torques on the body that can be calculated from attitude rate information [21]. The yaw angle to the relative wind can then be recovered from the aerodynamic moment database. Finally, the crosswind is determined from the angle between the inertial velocity and relative wind direction.

Accelerometer data provide an independent solution by using the ratio of the horizontal acceleration to the axial acceleration. Again, from the aerodynamic force database, the yaw angle to the relative wind is determined. The vehicle attitude is known from the onboard guidance system, and so the yaw angle can be determined and then the crosswind. During a typical AB pass with a peak density of $30 \mathrm{~kg} / \mathrm{km}^{3}$, the drag acceleration reaches about $15 \mathrm{~mm} / \mathrm{s}^{2}$. The ODY (MRO) noise level of $0.08(0.005) \mathrm{mm} / \mathrm{s}$ is equivalent to a change in heading of about $0.005(0.0003) \mathrm{rad}$. This maps into a cross- or vertical-wind sensitivity of $20(1.5) \mathrm{m} / \mathrm{s}$. Clearly, there is significant potential in directly recovering winds from accelerometer data.

Unfortunately, these processes have random noise components and biases. One of the potential biases is due to errors in the location of the center of mass of the vehicle. Center of mass location errors directly bias the aerodynamic moment coefficients. Both methods depend on the aerodynamic database, and although the database may be adequate for mission design and operation, there is no guarantee that it is adequate for wind recovery. Further, the two approaches are not completely independent, because the dominant force is drag in free-molecular flow and moments and forces are linearly dependent. Few orbits get into the strong transitional flow regime in which the forces and torques are less dependent.

Nevertheless, aerodynamic torque data have been used to obtain a mean crosswind during MGS AB [21], and the results compare favorably with upper-atmospheric G $\overline{C M}$ predictions, even though there are potential biases due to the broken solar array. Odyssey provides a much more favorable opportunity to recover winds, and studies have been proposed to do wind comparisons with winds from thermospheric models [22]. A preliminary result is shown in Fig. 10.
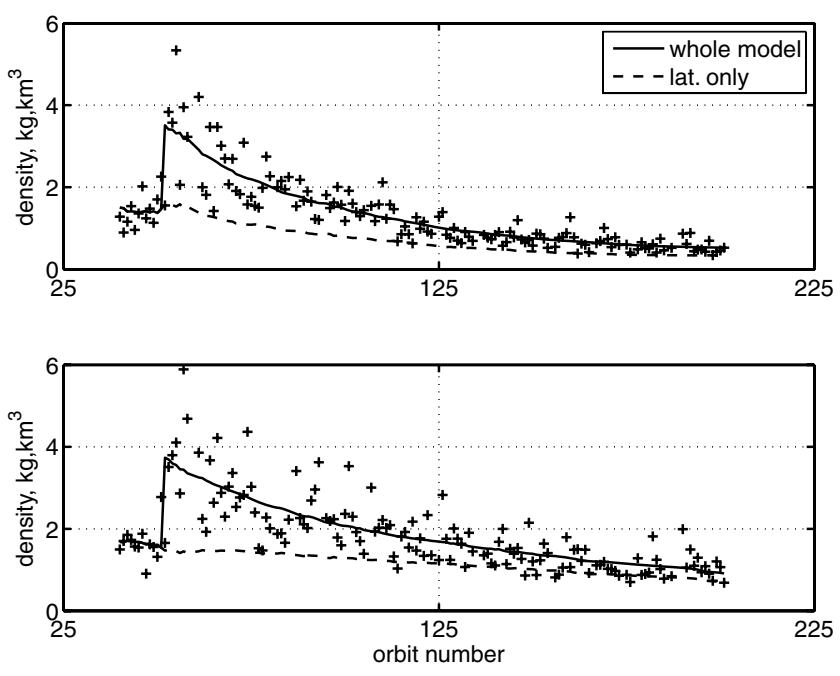

Fig. 9 Response of density at $140 \mathrm{~km}$ to the Noachis regional dust storm for both inbound (upper) and outbound (lower) legs.
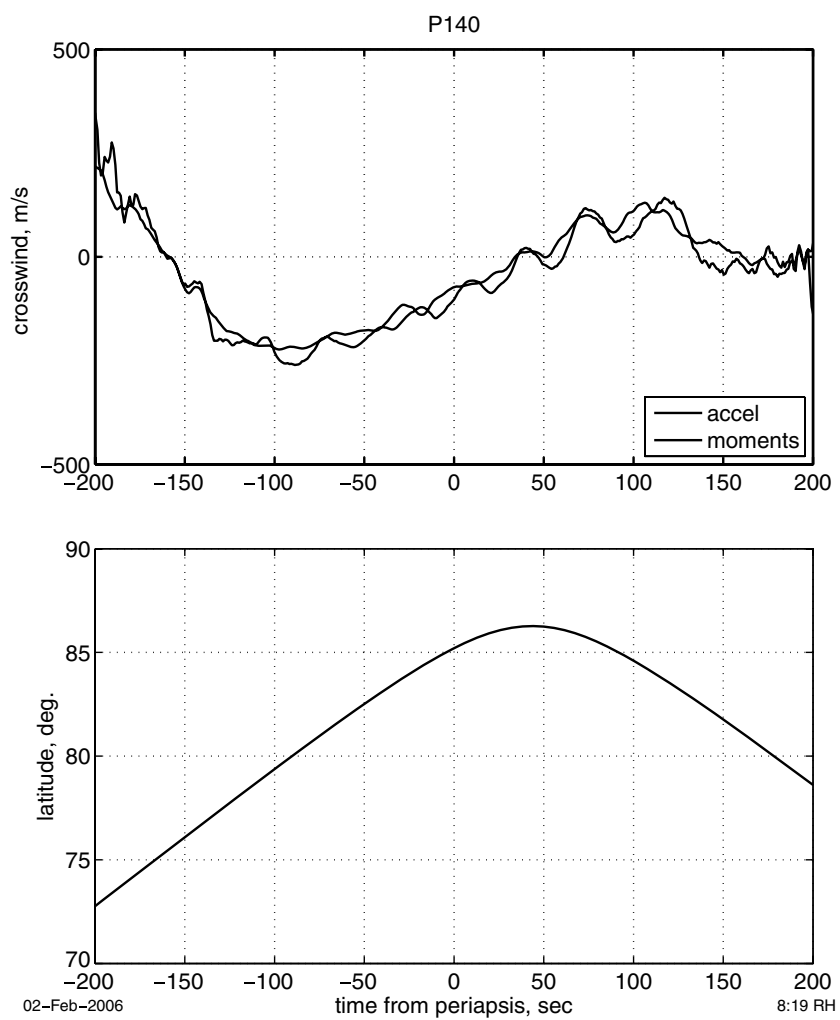

Fig. 10 Inferred crosswinds for ODY orbit 140.

The upper panel provides the recovered wind. One profile is derived from accelerometer data and the aerodynamic force database, and the second profile is derived from angular rate gyro data and the aerodynamic moment database. The overall trend in the winds is the same for either recovery. However, the vertical location of the moment derived winds is very sensitive to the location of the center of mass. Center of mass varies throughout the mission due to the use of attitude control fuel. Another example of engineering data required to recover atmospheric properties during $\mathrm{AB}$. The results in the figure were derived by solving for a fixed center of mass over a 20 -orbit interval. The vehicle passes closest to the pole at about $40 \mathrm{~s}$ after periapsis and the winds are nearly zero, as might be expected if the winds were purely zonal. This is not a consistent result through the full orbit set. For purely zonal winds, the crosswind changes direction in the spacecraft frame upon pole crossing, as shown in the 


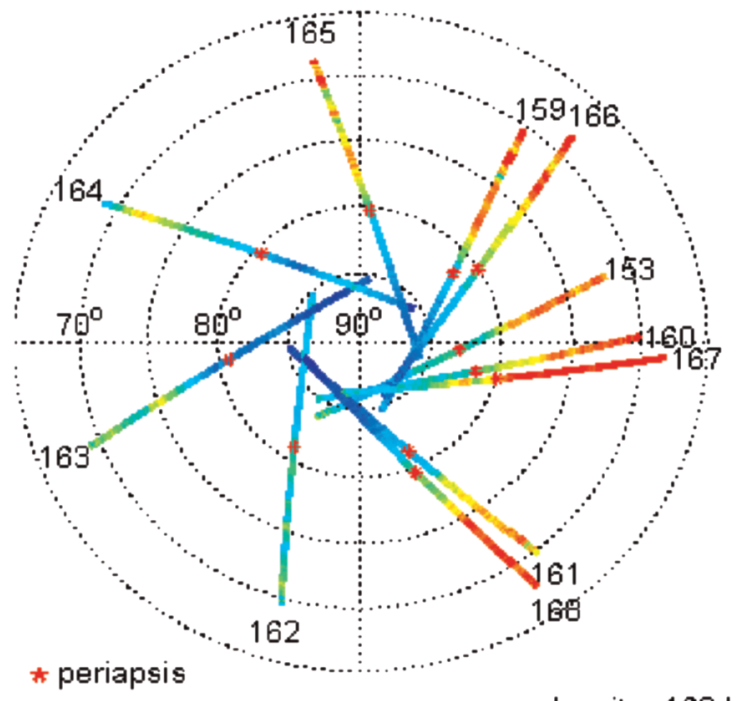

max density $=168 \mathrm{~kg}$

Fig. 11 Polar plot of densities mapped to $100-\mathrm{km}$ altitude for ODY orbits 153 and 159 through 168 . Color scale from 20 to $100 \mathrm{~kg} / \mathrm{km}^{3}$ and density truncated at $100 \mathrm{~kg} / \mathrm{km}^{3}$ to enhance variability.

figure. The winds are clearly stronger on the inbound leg than on the outbound, suggesting that the polar vortex is not symmetrical.

Additional evidence of the polar vortex and the asymmetry is shown in Fig. 11, taken from [7]. In an attempt to understand the temporal and spatial variations, polar plots of the density variation were made daily for orbits from the previous days. Each track on the plot shows the density along the orbit path on a latitude-longitude polar plot. All of the measured densities were mapped to a common reference altitude: in this case, $100 \mathrm{~km}$. Periapsis is identified by a star on each track. The time between close ground tracks (e.g., 160 and 167) is about 1 sol, and such adjacent pairs were compared to generate confidence in the mapping and to identify trends in the variations in the density field. A strong s-wave- 1 pattern is clearly evident at this time, with maximum density occurring in the first and fourth quadrants. Further, along-track variability is small within $10^{\circ}$ of the pole and increases as latitude moves south, consistent with Fig. 5. Along-track variability is most apparent in orbits 159, 165, and $\mathbf{1 6 6}$. The strong latitudinal density gradient away from the polar region is also quite evident, particularly in the first and fourth quadrants.

\section{Comparisons of Accelerometer Data}

Detailed discussions of accelerometer data processing are given in $[5,7,9]$ for the three missions. This section only summarizes some data noise considerations. The basic output of modern accelerometers is the change in velocity over the sample time, and so noise is quoted in change in velocity instead of acceleration. Depending on the implementation, sample rate may influence the conversion to acceleration accuracy. After orbit 18 of the MGS mission, the data noise and bias were essentially constant. The data noise was constant, because every sample (10/s) was transmitted and there was no preprocessing of the data on the vehicle or the ground. Bias was nearly constant, because the accelerometer block had an active temperature control system. Before the recognition of the broken solar array on orbit 15,10 contiguous counts of accelerometer data were transmitted every $8 \mathrm{~s}$. The profiles generated from these data produced much discussion about what we now recognize as the shortwave activity in the Martian thermosphere. Explanations ranged from instrument errors to $\mathrm{CO}_{2}$ sublimation off the solar arrays. During this trying time, the benefits of the accelerometer for engineering purposes became clear and more telemetry channels became available for the full data set when aerobraking resumed on orbit 40. Odyssey did not have actively thermal control of the IMU and showed bias shifts at about one-fifth of the noise level across an $\mathrm{AB}$ pass [7]. Odyssey accelerometer bias often increased rapidly at
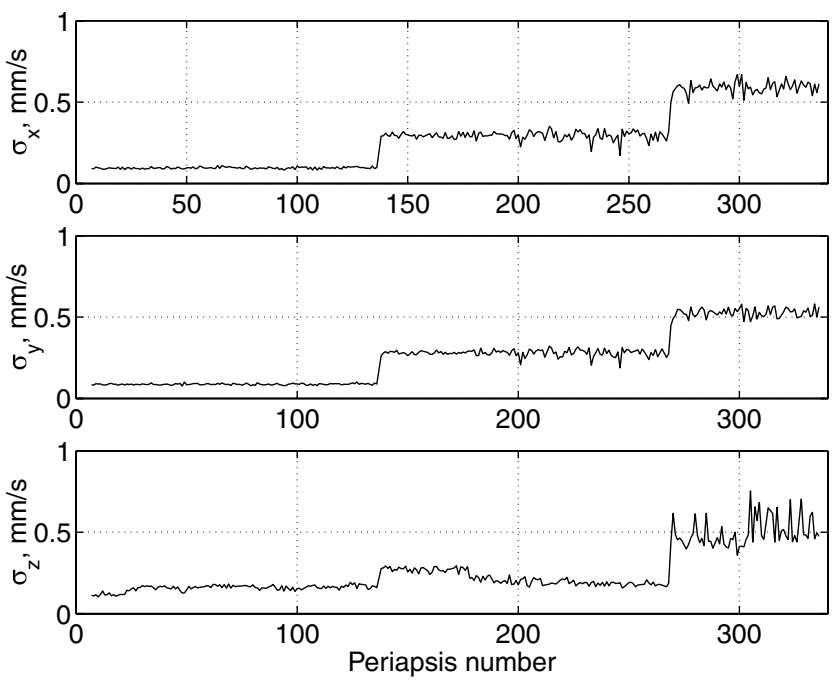

Fig. 12 Variation in accelerometer noise level throughout the ODY mission.

the end of the pass. The cause was likely aerodynamic heating of the bus; however, there were no IMU temperature measurements available with which to correlate. Further, there was some sort of double digitization inside the IMU that resulted in nonstationary noise. Because of onboard memory limitation, the sample size was reduced twice during the mission and, due to the sampling process, the coherence between the data was lost.

Figure 12 shows the noise level derived from data collected outside the atmosphere. For the first 138 orbits, the noise level of $0.086 \mathrm{~mm} / \mathrm{s}$ is fairly close to the least significant bit value of $0.0758 \mathrm{~mm} / \mathrm{s}$. The influence of changing the number of high rate samples from $200 /$ s to 50 and then to 20 is clearly evident. The lowest noise level corresponds to determining density to about $0.15 \mathrm{~kg} / \mathrm{km}^{3}$ and the largest corresponds to about $1.1 \mathrm{~kg} / \mathrm{km}^{3}$. This increase in noise level reduced the applicable altitude range of the data by two scale heights or between 15 to $20 \mathrm{~km}$. The higher noise level also occurred late in the mission when the periapsis altitude was being raised (Fig. 1), further reducing the value of the data. The MRO accelerometers are a modification of the models on ODY and have a noise level of between $2 \mathrm{e}-3$ and $5 \mathrm{e}-3 \mathrm{~mm} / \mathrm{s}^{2}$, or about 30 times smaller than the best ODY data. Based on noise level only, the sensitivity may be sufficient to measure density three scale heights higher. To accommodate the science data requirements, MRO had copious onboard memory and high-bandwidth communications. This provided large quantities of engineering data not seen on other missions. Such data included individual accelerometer temperature data, reaction control system catalyst bed temperatures, and other data that will help to improve the value of the accelerometer data to recover atmospheric properties. Nevertheless, as of this writing, there are unresolved issues that might degrade the minimum density recoverable. In particular, what appears to be fuel sloshing has clearly been identified in a number of orbital passes. Amplitudes as large as three times the noise level were found on the outbound leg at altitudes above $150 \mathrm{~km}$. Similar phenomena may have occurred on other missions, but were not observable due to the higher data noise. Modeling studies are underway to quantify this phenomena.

\section{Other Data Types}

The accelerometers are seldom mounted sufficiently close to the center of mass to ignore the accelerations due to the angular motion of the vehicle. During AB, the attitude control system is generally in the reaction control mode with a wide angular dead band and rate limited. Consequently, the body rates can become significant contributors to the measured acceleration. Off-the-shelf rate gyros generally have sufficient accuracy to correct the accelerometer measurements to the noise level, assuming the relative location of the 


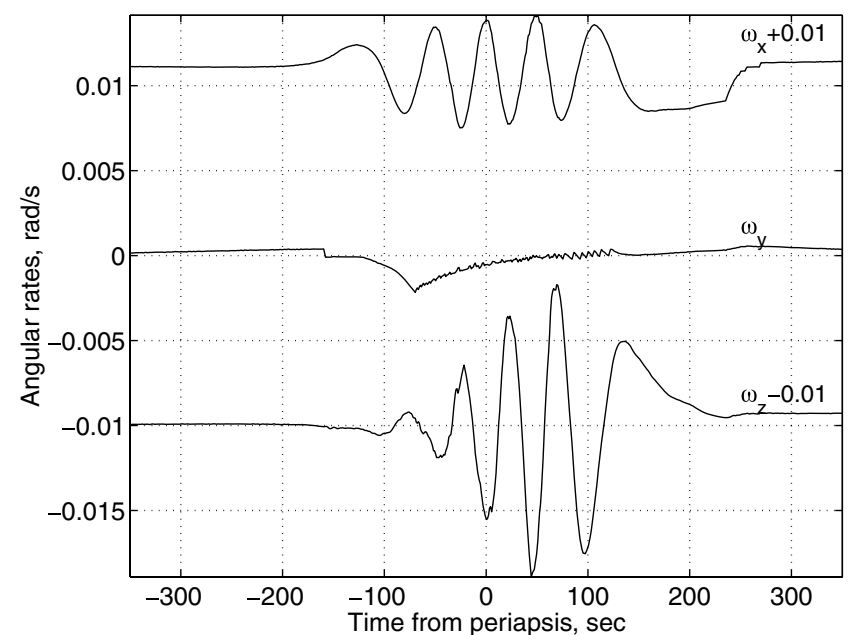

Fig. 13 Body angular rates during ODY P076, rates displaced for clarity.

accelerometers to the center of mass is accurately estimated preflight or calibrated in flight.

A typical history of the body rates for ODY are shown in Fig. 13. The $x(z)$ rates are shifted up (down) for clarity. The $y$ axis is into the wind and had no aerodynamic restoring force. The other two axes are stable. Motion about all three axes are coupled through the cross products of inertia and onboard momentum supplied by the reaction wheels. The angular acceleration derived from these rates is the major contributor to the correction of the measured acceleration. For typical $\mathrm{AB}$ passes, the maximum contribution is less than $0.5 \mathrm{~mm} / \mathrm{s}^{2}$, which is larger than the noise level. From the $y$ axis angular rate data, it is seen that large thruster firings took place at -160 and $-75 \mathrm{~s}$. Though more difficult to see, there are nearly continuous small firings from $-75 \mathrm{~s}$ through about $120 \mathrm{~s}$. These particular attitude control jets are coupled and theoretically produce no net acceleration of the $s / c$. However, residual imbalances remain and short impulses are very difficult to calibrate due to the sensitivity of the total impulse to initial catalyst temperatures and other parameters. For MRO, the minimum attitude thruster firing introduces a change in velocity about three times the noise level on the accelerometer data. MRO telemetry contains temperature data for each accelerometer and the thruster catalyst bed temperature. Postflight analysis will determine the value of these data in improving accelerometer data quality.

\section{Lessons Learned}

A well-designed experiment to recover atmospheric density requires numerous supplemental data sets. Further, a systems approach to the overall design of the vehicle is required to extract the most information from the data. For example, potential degradation of the accelerometer data can result from structural vibration or fuel slosh. The broken solar array on MGS introduced a $6-7-\mathrm{Hz}$ oscillation, and the archived data set is a seven-point mean of the 1-s density recoveries. This vibration degraded the potential horizontal spatial resolution from 4 to $25 \mathrm{~km}$. Generally, structural vibration is sufficiently small and of high enough frequency to have a negligible influence on the density recovery. Fuel slosh is a different issue. When out of the atmosphere, the fuel distribution in the tanks is controlled by surface tension. As the vehicle enters the atmosphere, drag eventually overcomes surface tension and the fuel moves forward. The center of mass of the vehicle moves relative to the accelerometers and produces an artifact in the data. It is thought that any oscillation is rapidly damped because drag is rapidly increasing on the inbound leg. On exiting the atmosphere, the process is reversed, but now the fuel can slosh around under the weak surface tension forces. Slosh oscillation periods are difficult to estimate, but appear to be on the order of 25 to $100 \mathrm{~s}$ and could introduce artifacts that might be misinterpreted as density waves in the upper atmosphere.
Geometric symmetry and simplicity are also highly desirable for density or wind recovery. The fidelity of the aerodynamic database is likely diminished for asymmetric bodies or bodies that have complicated geometries. During the inbound leg, the dynamic pressure increases and attitude oscillations are damped. After maximum dynamic pressure is reached, the attitude oscillations rapidly increase and the vehicles often experience large yaw or pitch angles. Vehicles with complicated geometries make accurate aerodynamic calculation more difficult at these high angles. For a dedicated aeronomy mission that depends on accelerometer data, a system study should be performed to trade tighter attitude rate control, thruster torque unbalance, attitude fuel usage, and aerodynamic database accuracy to optimize the science return.

\section{Conclusions}

MGS was the first Mars mission in which an accelerometer experiment was project-approved. The experiment was not an official science experiment and had none of the privileges given to such experiments. When aerobraking began, of the 10 samples per second collected onboard the vehicle, two samples were transmitted each second and all 10 were sent every $8 \mathrm{~s}$. There was not enough telemetry bandwidth to include more accelerometer data, because of all the other engineering data required to monitor the health of the spacecraft. The two-sample-per-second data proved useless, but the full sample every $8 \mathrm{~s}$ provided reasonable profiles and showed curious features, one being called "Batman's ears." On orbit 15, the solar array deflected $10 \mathrm{deg}$ from its nominal position and the project realized it had a major anomaly. After aerobraking restarted 20 orbits later, all ten samples were sent every second, because one way of monitoring the vibration of the solar array was with the accelerometer data. This serendipitous data set led to discoveries of the planetary scale waves, the gravity waves, and the incredible latitudinal, seasonal, and diurnal variability of the atmosphere. These data still provide the basis for numerous ongoing scientific studies. So there was at least one good outcome from the broken solar array.

The great variability of the atmosphere, discovered by MGS, added a level of risk to future $\mathrm{AB}$ missions. Consequently, real-time analysis of accelerometer measurements during $\mathrm{AB}$ operations became a high priority for ODY. Because the accuracy of the accelerometers was adequate to monitor aerobraking, the accelerometer design would not be enhanced for scientific studies. Nevertheless, ODY accelerometer measurements provided additional insight into the Martian thermosphere. The scientific benefits of these data became generally accepted, and MRO was the first mission to include the accelerometer as a facility experiment, with a competition for selection of the facility team. With that status, the team could place measurement requirements on the project. This resulted in the significantly improved accuracy of MRO over ODY that resulted from modification of the ODY accelerometer electronics.

The potential exists to use accelerometer data to make additional contributions to our knowledge of the upper atmosphere. Higher spatial resolution could provide information on gravity wave dissipation processes, greater sensitivity would permit higheraltitude measurements and could quantify the transition from the thermosphere to the exosphere, and using an orbit with a lower inclination would provide in situ longitudinal and diurnal structure. To assure success of future accelerometer experiments requires that the accelerometer be recognized as a scientific instrument and be given the same role in the spacecraft and mission design as is currently afforded to a camera or a spectrometer.

\section{Acknowledgments}

The authors would like to acknowledge NASA support through the Mars Data Analysis program and Jet Propulsion Laboratory, California Institute of Technology support during the aerobraking operational phases of Mars Global Surveyor, Odyssey, and Mars Reconnaissance Orbiter. 


\section{References}

[1] Lyons, D., "Aerobraking Magellan: Plan Versus Reality," Spaceftight Mechanics 1994, Advances in Astronautical Sciences, Vol. 87, Pt. 2, Univelt, San Diego, CA, for AIAA, Reston, VA, 1994, pp. 663680.

[2] Keating, G. M., Hsu, N. C., and Lyu, J., "Improved Thermospheric Model for the Venus International Reference Atmosphere," Advances in Space Research, Vol. 19, No. 8, May 1997, p. 1292.

[3] Croom, C., and Tolson, R., "Using Magellan Attitude Control Data to Study the Venusian Atmosphere and Various Spacecraft Properties," AAS/AIAA Spaceflight Mechanics Meeting, American Astronautical Society, Paper 94-141, 14-16 Feb. 1994.

[4] Espiritu, R., and Tolson, R., "Determining Venusian Upper Atmosphere Characteristics Using Magellan Attitude Control Data," AIAA/AAS Spaceflight Mechanics Meeting, Alburquerque, NM, American Astronautical Society, Paper 95-152, 13-16 Feb. 1995.

[5] Tolson, R. H., Keating, G. M., Cancro, G. J., Parker, J. S., Noll, S. N., and Wilkerson, B. L., "Application of Accelerometer Data to Mars Global Surveyor Aerobraking Operations," Journal of Spacecraft and Rockets, Vol. 36, No. 3, 1999, pp. 323-329.

[6] Tolson, R., Keating, G., Noll, S., Baird, D., and Shellenberg, T., "Utilization of Mars Global Surveyor Accelerometer Data for Atmospheric Modeling," Astrodynamics 1999, Advances in Astronautical Sciences, Vol 103, Pt. 2, Univelt, San Diego, CA, for AIAA, Reston, VA, 2000, pp. 1329-1346.

[7] Tolson, R., Dwyer, A. M., Hanna, J. L., Keating, G. M., George, B. E., Escalera, P. E., and Werner, M. R., "Application of Accelerometer Data to Mars Odyssey Aerobraking and Atmospheric Modeling," Journal of Spacecraft and Rockets, Vol. 42, No. 3, 2005, pp. 435-443.

[8] Graf, J., Zurek, R., Jones, J., Eisen, H., Johnson, M., and Jai, B., "An Overview of the Mars Reconnaissance Orbiter Mission," 2002 IEEE Aerospace Conference Proceedings, Vol. 1, 11-15 Mar. 2002, pp. 1180.

[9] Tolson, R., Bemis, E., Hough, S., Zaleski, K., Keating, G., Shidner, J., Brown, S., Brickler, A., Scher, M., and Thomas, P., "Atmospheric Modeling Using Accelerometer Data During Mars Reconnaissance Orbiter Aerobraking Operations," AAS/AIAA Spaceflight Mechanics Meeting, American Astronautical Society, Paper 07-183, 28 Jan.01 Feb. 2007.

[10] Justus, C., James, B., Bougher, S., Bridger, A., Haberle, R., Murphy, J., and Engel, S., "Mars-GRAM 2000: A Mars Atmospheric Model for Engineering Applications," Advances in Space Research, Vol. 29, No. 2, 2002, pp. 193-202. doi:10.1016/S0273-1177(01)00569-5

[11] Justus, C., Duvall, A., and Johnson, D., "Mars Global Reference Atmospheric Model (MARS-GRAM) and Database for Mission Design," International Workshop on Mars Atmosphere Modelling and Observations, Grenada, Spain, Centre National d'Etudes Spatiales and ESA, Paper 17-1, 13-15 Jan. 2003.

[12] Smith, J., and Bell, J., "2001 Mars Odyssey Aerobraking," AIAA/AAS Astrodynamics Specialist Conference, AIAA Paper 2002-4532, 58 Aug. 2002.
[13] Bougher, S., Bell, J., Murphy, J., Lopez-Valverde, M., and Withers, P., "Polar Warming in the Mars Thermosphere: Seasonal Variations Owing to Changing Insolation and Dust Distributions," Geophysical Research Letters, Vol. 33, 2006, p. L02203. doi:10.1029/2005GL024059

[14] Fritts, D. C., Wang, L., and Tolson, R. H., "Mean and Gravity Wave Structures and Variability in the Mars Upper Atmosphere Inferred from MGS And MO Aero-Braking Densities," Journal of Geophysical Research, Vol. 111, 2006, p. A12304. doi:10.1029/2006JA011897

[15] Creasey, J. E., Forbes, J. M., and Keating, G. M., "Density Variability at Scales Typical of Gravity Waves Observed in Mars' Thermosphere by the MGS Accelerometer," Geophysical Research Letters, Vol. 33, 2006, p. L22814. doi:10.1029/2006GL027583

[16] Dwyer, A., Tolson, R., Monk, M., and Tartabini, P., "Development of a Monte Carlo Mars-GRAM Model for Mars 2001 Aerobraking Simulations," Astrodynamics 2001, Advances in Astronautical Sciences, Vol. 109, Univelt, San Diego, CA, for AIAA, Reston, VA, 2002, pp. 1293-1308.

[17] Keating, G. M., Bougher, S. W., Zurek, R. W., Tolson, R. H., Cancro, G. J., Noll, S. N., Parker, J. S., Schellenberg, T. J., Shane, R. W., Wilkerson, B. L., Murphy, J. R., Hollingsworth, J. L., Haberle, R. M., Joshi, M., Pearl, J. C., Conrath, B. J., Smith, M. D., Clancy, R. T., Blanchard, R. C., Wilmoth, R. G., Rault, D. F., Martin, T. Z., Lyons, D. T., Esposito, P. B., Johnston, M. D., Whetzel, C. W., Justus, C. G., and Babicke, J. M., "The Structure of the Upper Atmosphere of Mars: In Situ Accelerometer Measurements from Mars Global Surveyor ," Science, Vol. 279, No. 5357, 1998, pp. 1672-1679. doi: $10.1126 /$ science. 279.5357 .1672

[18] Wilson, R., and Hamilton, K., "Comprehensive Model Simulation of Thermal Tides in the Martian Atmosphere," Journal of Atmospheric Sciences, Vol. 53, No. 9, 1996, pp. 1290-1326. doi:10.1175/1520-0469(1996)053<1290:CMSOTT>2.0.CO;2

[19] Forbes, J. M., , Bridger, A. F. C., Bougher, S. W., Hagan, M. E., Hollingsworth, J. L., Keating, G. M., and Murphy, J., "Nonmigrating Tides in the Thermosphere of Mars," Journal of Geophysical Research, Vol. 107, No. E11, 2002, p. 5113. doi:10.1029/2001JE001582

[20] Wilson, R., "Evidence for Nonmigrating Thermal Tides in the Mars Upper Atmosphere from the Mars Global Surveyor Accelerometer Experiment," Geophysical Research Letters, Vol. 29, No. 7, 2002, p. 1120. doi:10.1029/2001GL013975

[21] Baird, D., Tolson, R., and Bougher, S., "Calculation of Zonal Winds Using Accelerometer and Rate Data from Mars Global Surveyor," AIAA Paper 2006-6390, Aug. 2006.

[22] Crowley, G., and Tolson, R., "Mars Thermospheric Winds from MGS and Odyssey Accelerometers," AIAA Paper 2006-6392, Aug. 2006.

A. Ketsdever Associate Editor 